Original Article

\title{
Ethnomedicinal and traditional uses of the Ferns of Khyber Pakhtunkhwa, Pakistan
}

\author{
Usos etnomedicinais e tradicionais das samambaias de Khyber Pakhtunkhwa, \\ Paquistão
}

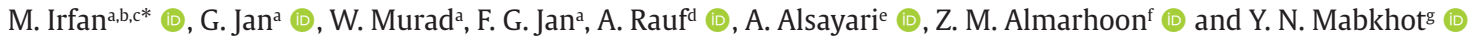 \\ aAbdul Wali Khan University, Department of Botany, Mardan, Pakistan \\ bUniversity of Swabi, Department of Botany, Swabi, Pakistan \\ 'Missouri Botanical Garden, St. Louis, Missouri, U.S.A \\ dUniversity of Swabi, Department of Chemistry, Swabi, Pakistan \\ eKing Khalid University, College of Pharmacy, Department of Pharmacognosy, Abha, Saudi Arabia \\ fKing Saud University, College of Science, Department of Chemistry, Riyadh, Saudi Arabia \\ ${ }^{\text {g} K i n g ~ K h a l i d ~ U n i v e r s i t y, ~ C o l l e g e ~ o f ~ P h a r m a c y, ~ D e p a r t m e n t ~ o f ~ P h a r m a c e u t i c a l ~ C h e m i s t r y, ~ A b h a, ~ S a u d i ~ A r a b i a ~}$
}

\begin{abstract}
Ferns are often used by indigenous people in Khyber Pakhtunkhwa, Pakistan. This study was designed to collect the ethnomedicinal and traditional knowledge of these locals about this group of vascular plants. Forty taxa belong to nineteen genera and ten families are used in the treatment of different diseases. The Pteridaceae was the most representative family with twelve taxa (30\%), followed by Athyriaceae and Dryopteridaceae with six taxa each (30\%), and Thelypteridaceae with five taxa (12.5\%). Regarding the genera, Adiantum, Asplenium and Dryopteris ranked first with four taxa each (30\%), followed by Aleuritopteris, Diplazium, Pteris and Equisetum with three taxa each (30\%), followed by Athyrium, Oeosporangium, Polystichum and Pseudophegopteris with two taxa each (20\%). These taxa were commonly used in the treatment of respiratory disorders i.e. asthma, bronchitis, emphysema, pneumonia; intestinal ulcer, stomach, urinary ailments and skin disorders by the methods of decoction and infusion. Traditional knowledge about ethnomedicinal plants is a valuable and essential source for the discovery of allopathic, herbal and homeopathic medicines.
\end{abstract}

Keywords: ethnomedicinal, ferns, Khyber Pakhtunkhwa, Pakistan, traditional.

\begin{abstract}
Resumo
As samambaias são frequentemente usadas pelos indígenas em Khyber Pakhtunkhwa, Paquistão. Este estudo foi desenhado para coletar o conhecimento etnomedicinal e tradicional desses moradores sobre este grupo de plantas vasculares. Quarenta táxons pertencem a dezenove gêneros, e dez famílias são utilizadas no tratamento de diferentes doenças. Pteridaceae foi a família mais representativa com doze táxons (30\%), seguida por Athyriaceae e Dryopteridaceae com seis táxons cada (30\%), e Thelypteridaceae com cinco táxons (12,5\%). Em relação aos gêneros, Adiantum, Asplenium e Dryopteris ficaram em primeiro lugar com quatro táxons cada (30\%), seguidos por Aleuritopteris, Diplazium, Pteris e Equisetum com três táxons cada (30\%), e Athyrium, Oeosporangium, Polystichum e Pseudophegopteris com dois táxons cada (20\%). Estes táxons foram comumente usados no tratamento de distúrbios respiratórios, isto é, asma, bronquite, enfisema, pneumonia; úlcera intestinal, estômago, doenças urinárias e doenças da pele pelos métodos de decocção e infusão. O conhecimento tradicional sobre plantas etnomedicinais é uma fonte valiosa e essencial para a descoberta de medicamentos alopáticos, fitoterápicos e homeopáticos.
\end{abstract}

Palavras-chave: etnomedicinal, ferns, Khyber Pakhtunkhwa, Paquistão, tradicional.

\section{Introduction}

All living organisms depend upon food and medicines (Irfan et al., 2017a, b). Plants are ethnomedicinally used in different communities of the world since centuries ago (Irfan et al., 2018a; Ullah et al., 2018). Now a day's modern medicines are replacing the ethnomedicinal uses of the plants around the world (Iftikhar et al., 2019; Irfan et al., 2018b, 2019). Until now 70\% inhabitants around the world depends upon the ethnomedicinal uses of plants (Irfan et al., 2018c). Most of the population of poor and developing countries relies upon traditional medicines (Attaullah et al., 2017; Irfan et al., 2018d; Singh and Upadhyay, 2014). In Pakistan about 700 plant species are

*e-mail: Mirfan310@yahoo.com

Received: 25 Mar. 2021 - Accepted: 22 Sep. 2021 
medicinally used in rural and tribal areas due to high cost on modern medicines and poverty (Ahmad et al., 2016; Irfan et al., 2018e).

Worldwide there are about 11916 taxa with 337 genera, 51 families, 14 orders and two classes of ferns and lycophytes (PPG-I, 2016). A total of 168 taxa with 45 genera and 19 families are currently reported from Pakistan with greater wealth in Azad Jammu and Kashmir, Khyber Pakhtunkhwa and Gilgit-Baltistan, Pakistan (FraserJenkins et al., 2016, 2018, 2020; Irfan et al., 2021d).

Ferns have been used as prophylactic measure in different respiratory, urinary and skin disorders (Mannan et al., 2008; Nair, 1959; Kaushik and Dhiman, 1995; Gul et al., 2016b). New plant taxa have been added to the Flora of Pakistan having great medicinal importance (Ali et al., 2017). First time ethnomedicinal uses of ferns were reported in Jammu and Kashmir, India (May, 1978). Sixty-six species representatives of this group of vascular plants were reported from India for the treatment of various ailments (Kumar et al., 2003). Ferns were ethnomedicinally used for the treatment of various diseases, such as ulcer, urinary infections, vomiting, hair fall, sterility, dysentery and healing of wounds (Keller et al., 2011; Keller and Prance, 2015; Liu et al., 2012; Oloyede et al., 2008; Ranil and Bussmann, 2021). Ethnomedicinal uses of different ferns species have been documented by many workers, from Western Ghats, India (Benjamin and Manickkam, 2007); Arunachal Pradesh, North Eastern, India (Benniamin, 2011); Kumaun Himalaya, Uttarakhand, India (Upreti et al., 2009); Vindhyan Region (M.P.), India (Pathak et al., 2011); Kolli hills, Namakkal District, Tamil Nadu, India (Perumal, 2010); Similipal biosphere reserve, Orissa, India (Rout et al., 2009); Kerala, South India (Kumar et al., 2003); Rajasthan, India (Parihar and Parihar, 2006); Banajalaya conserved forest area of Shimoga District, Karnataka, India (Deepa et al., 2014) and from others mainland of India (Singh and Viswanathan, 1996; Vasudev, 1999). Ferns are used as a source of food, fibers, fuel (Biplab and Subir, 2007); screen heavy metals from the soil (Deepa et al., 2014); enhance environmental beauty and reduces pollution (Ranil et al., 2015; Gul et al., 2016b); adds pleasant scenery of gardens, parks and houses (Manickam and Irudayaraj, 2003); improves environmental beauty of offices, schools and hospitals (Mittal and Bir, 2006; Pande, 1991).

The major ferns genera dominated in Khyber Pakhtunkhwa, Pakistan are Asplenium, Dryopteris, Polystichum, Athyrium, Pteris, Adiantum, Aleuritopteris, Cystopteris, Selaginella, Diplazium, Equisetum, Oeosporangium, Onychium, Cryptogramma, Notholaena, Hypodematium, Pteridium, Marsilea, Anogramma, Actiniopteris, Dennstaedtia and Gymnocarpium (Fraser-Jenkins, 1992, 2014; Gul et al., 2016a). Due to variable climatic, topographic, edaphic and physiographic features with highest humidity and precipitation in higher elevations Khyber Pakhtunkhwa occupies greater wealth of lycophytes and ferns (Irfan et al., 2021a, b, c).

\section{Materials and Methods}

Ethnomedical and traditional uses of the ferns in Khyber Pakhtunkhwa were investigated through questionnaire method from the local inhabitants; herbal practitioners, Hakeem's, medicinal plants traders, wound healers and aged women from March 2018 to August 2019. A total of 150 questionnaires were conducted from informers at different districts of Khyber Pakhtunkhwa, Pakistan, i.e. Abbottabad, Buner, Chitral, Dir Lower, Dir Upper, Khyber, Kurram, Malakand, Mansehra, Swabi, Swat, Shangla and Torghar. During field trips the potential indigenous knowledge about its use were documented. Plant specimens were collected, photographed, tagged, pressed, preserved and mounted on exsiccates and later deposited at the herbarium of Abdul Wali Khan University, Mardan, Khyber Pakhtunkhwa, Pakistan. Plant specimens were identified by Mr. C. R. Fraser-Jenkins and were classified according to (PPG-I, 2016).

Fidelity level (FL) was calculated according to Equation 1 (Alexiades, 1996).

$$
\operatorname{FL}(\%)=\operatorname{Ip} / \mathrm{Iu} 100
$$

The fidelity level is the percentage of respondents mentioning the uses of a specific plant to treat particular disease. Ip is the number of informants independently suggested the use of various taxa for a specific disorder, while Iu is the total number of informants suggested various taxa for the ethnomedicinal purpose.

Use value (UV) was calculated according to Equation 2 (Phillips et al., 1994).

$$
\mathbf{U V i}=\Sigma \mathbf{U i} / \mathbf{N i}
$$

Use value (UV) is a numerical method that proves the relative importance regarding medicinal uses of plant species; UV is the number of individual use species, $\mathrm{Ui}$ is the number of uses recorded for each species and $\mathrm{Ni}$ is the total number of informants. Use value reflects the relative importance of reported plant species in an area. High use value shows that plant species have many use reports and is important in the region, whereas low use value (approach to 0 ) shows that species have few use reports related to its use. However, use report is not meaningful to differentiate whether a plant species is used for single or manifold purposes (Kayani et al., 2014).

Relative frequency was calculated according to Equation 3 (Ali-Shtayeh et al., 2016).

$$
\mathbf{R F C}=\mathbf{F C} / \mathbf{N}(\mathbf{0}<\mathbf{R F C}<\mathbf{1})
$$

Relative frequency of citation (RFC) presents the local importance of each species in a study area. To calculate RFC, number of respondents citing a useful species (FC) is divided by total number of respondents in the field survey (N). RFC value varies from 1 (when all the respondents refer to a plant as a useful one) to 0 (when nobody refers to a plant as a valuable species). FC is the number of informants using the plant species, $\mathrm{N}$ is the total number of informants (Ahmad et al., 2014).

Jaccard index (JI) was calculated according to Equation 4 (González-Tejero et al., 2008). 


$$
\mathbf{J I}=\mathbf{c} \times \mathbf{1 0 0} \div(\mathbf{a}+\mathbf{b}-\mathbf{c})
$$

"a" is the total number of taxa used in Khyber Pakhtunkhwa, Pakistan, "b" is the total number of taxa previously published in each article from India, while "c" is the total number of taxa common to both $\mathrm{A}$ and $\mathrm{B}$.

The similarity of knowledge between different communities was determined by comparing the findings of the current study with already published articles from India by applying Jaccard index. The studies conducted on the areas with similar, vegetation, climatic condition, and culture was considered for comparison.

\section{Results}

The current research found 40 taxa of ferns belongs to 19 genera and 10 families with ethnomedicinal and traditional used in the prophylaxis of different ailments (Table 1). Amongst 10 ferns plant families Pteridaceae was

Table 1. Ethnomedicinal and traditional uses of the ferns of Khyber Pakhtunkhwa, Pakistan.

\begin{tabular}{|c|c|c|c|c|c|c|c|}
\hline Taxon & Family & $\begin{array}{l}\text { Vernacular } \\
\text { name }\end{array}$ & $\begin{array}{c}\text { Part } / \mathbf{s} \\
\text { used }\end{array}$ & $\begin{array}{c}\text { Method of application and Disease } \\
\text { cured }\end{array}$ & UV & FL & RFC \\
\hline $\begin{array}{l}\text { Asplenium } \\
\text { adiantum- } \\
\text { nigrum } \text { L. subsp. } \\
\text { adiantum-nigrum }\end{array}$ & Aspleniaceae & Sumbal & Yl & $\begin{array}{l}\text { Ten grams extract of young leaves used } \\
\text { orally for treatment of chest infections, } \\
\text { i.e. asthma, bronchitis and pneumonia. }\end{array}$ & 0.1 & 0.13 & 0.35 \\
\hline $\begin{array}{l}\text { Asplenium } \\
\text { ceterach } \text { L. subsp. } \\
\text { ceterach }\end{array}$ & Aspleniaceae & Naroky & Yl, R & $\begin{array}{l}\text { Young leaves extract used against } \\
\text { enema in children. Women use rhizome } \\
\text { decoction against miscarriage and } \\
\text { infertility. }\end{array}$ & 0.06 & 0.4 & 0.29 \\
\hline $\begin{array}{l}\text { Asplenium } \\
\text { dalhousiae Hook. }\end{array}$ & Aspleniaceae & Naroky & Yl & $\begin{array}{l}\text { Decoction of young leaves used orally for } \\
\text { ten days against hepatitis having anti- } \\
\text { viral effects. }\end{array}$ & 0.09 & 0.6 & 0.21 \\
\hline $\begin{array}{l}\text { Asplenium } \\
\text { trichomanes } \\
\text { L. subsp. } \\
\text { trichomanes }\end{array}$ & Aspleniaceae & Jenabil & $\mathrm{Yl}$ & $\begin{array}{l}\text { Fifty grams leaves boiled mixed with } \\
\text { coconut oil applied on skin for treatment } \\
\text { of skin infections, i.e. leukoderma and } \\
\text { psoriasis. }\end{array}$ & 0.15 & 0.26 & 0.25 \\
\hline $\begin{array}{l}\text { Athyrium } \\
\text { atkinsonii Bedd. }\end{array}$ & Athyriaceae & Mangeti & Yl & $\begin{array}{l}\text { Decoction of leaves mixed with gum } \\
\text { Acacia powder used as an herbal tonic } \\
\text { against body weakness. }\end{array}$ & 0.9 & 0.5 & 0.21 \\
\hline $\begin{array}{l}\text { Athyrium } \\
\text { schimperi Moug. } \\
\text { ex Fée subsp. } \\
\text { biserrulatum } \\
\text { (Christ) Fraser- } \\
\text { Jenk. }\end{array}$ & Athyriaceae & Mangeti & Yl & $\begin{array}{l}\text { Ten ml young leaves extract used to } \\
\text { prevent ulcer and intestinal disorders. }\end{array}$ & 0.3 & 0.1 & 0.25 \\
\hline $\begin{array}{l}\text { Cystopteris fragilis } \\
\text { (L.) Bernh. subsp. } \\
\text { fragilis }\end{array}$ & Athyriaceae & Kandad & $\mathrm{Yl}$ & $\begin{array}{l}\text { Paste of young leaves mixed with } \\
\text { mustard oil applied on hairs against } \\
\text { dandruff, hair fall and maintains hair } \\
\text { shine. }\end{array}$ & 0.1 & 0.3 & 0.29 \\
\hline $\begin{array}{l}\text { Diplazium } \\
\text { esculentum } \\
\text { (Retz.) Sw }\end{array}$ & Athyriaceae & Kunji saag & Yl & $\begin{array}{l}\text { Young leaves palatable used as vegetable } \\
\text { enriched with iron reduces iron } \\
\text { deficiency and is diuretic. }\end{array}$ & 0.4 & 0.6 & 0.32 \\
\hline $\begin{array}{l}\text { Diplazium } \\
\text { longifolium } \\
\text { (D.Don) T.Moore }\end{array}$ & Athyriaceae & Kunji saag & Yl & $\begin{array}{l}\text { Young leaves palatable used as vegetable } \\
\text { enriched with iron and is purgative. }\end{array}$ & 0.6 & 0.5 & 0.5 \\
\hline $\begin{array}{l}\text { Diplazium } \\
\text { maximum } \\
\text { (D.Don) C.Chr. }\end{array}$ & Athyriaceae & Kunji & $\mathrm{R}$ & $\begin{array}{l}\text { Decoction of rhizome used empty } \\
\text { stomach removes intestinal worms. }\end{array}$ & 0.6 & 0.33 & 0.6 \\
\hline $\begin{array}{l}\text { Woodwardia } \\
\text { unigemmata } \\
\text { (Makino) Nakai }\end{array}$ & Blechnaceae & Banjasa & $\mathrm{R}, \mathrm{Yf}$ & $\begin{array}{l}\text { Decoction of rhizome used orally at night } \\
\text { after meal causes expulsion of intestinal } \\
\text { worms. Infusion of young frond used } \\
\text { to relieve stomach cramps and increase } \\
\text { urine flow. }\end{array}$ & 0.2 & 0.2 & 0.1 \\
\hline
\end{tabular}

Key for part used: C: Cones; R: Rhizome; WP: Whole plant; Yf: Young frond; Yl: Young leaves. 
Table 1. Continued...

\begin{tabular}{|c|c|c|c|c|c|c|c|}
\hline Taxon & Family & $\begin{array}{l}\text { Vernacular } \\
\text { name }\end{array}$ & $\begin{array}{c}\text { Part } / \mathbf{s} \\
\text { used }\end{array}$ & $\begin{array}{c}\text { Method of application and Disease } \\
\text { cured }\end{array}$ & UV & $\mathbf{F L}$ & RFC \\
\hline $\begin{array}{l}\text { Dryopteris } \\
\text { blanfordii } \\
\text { (C.Hope) C.Chr. } \\
\text { subsp. blanfordii }\end{array}$ & Dryopteridaceae & Mangeti & Yf & $\begin{array}{l}\text { Paste of whole plant applied on skin } \\
\text { against snake, scorpion and insect bites. }\end{array}$ & 0.04 & 0.2 & 0.5 \\
\hline $\begin{array}{l}\text { Dryopteris } \\
\text { nigropaleacea } \\
\text { (Fraser-Jenk.) } \\
\text { Fraser-Jenk. }\end{array}$ & Dryopteridaceae & Ratanjot & Yf, Yl & $\begin{array}{l}\text { Fronds removes ghosts and unseen evils, } \\
\text { leaves extract mixed with coconut oil } \\
\text { applied on body to cure skin disorders, } \\
\text { i.e. urticaria and sarcoma. }\end{array}$ & 0.06 & 0.66 & 0.29 \\
\hline $\begin{array}{l}\text { Dryopteris } \\
\text { redactopinnata } \\
\text { S.K.Basu \& } \\
\text { Panigrahi }\end{array}$ & Dryopteridaceae & Mangeti & $\mathrm{R}$ & $\begin{array}{l}\text { Paste of rhizome mixed with olive oil } \\
\text { used to cure eczema and stomachache. }\end{array}$ & 0.08 & 0.46 & 0.41 \\
\hline $\begin{array}{l}\text { Dryopteris } \\
\text { stewartii Fraser- } \\
\text { Jenk. }\end{array}$ & Dryopteridaceae & Mangeti & $\mathrm{R}$ & $\begin{array}{l}\text { Decoction of rhizome used for twenty } \\
\text { days orally against rheumatism. }\end{array}$ & 0.09 & 0.33 & 0.61 \\
\hline $\begin{array}{l}\text { Polystichum } \\
\text { piceopaleaceum } \\
\text { Tagawa }\end{array}$ & Dryopteridaceae & Kunji & Yf & $\begin{array}{l}\text { Rhizome powder mixed with coconut } \\
\text { oil and sugar used orally for healing of } \\
\text { wounds. }\end{array}$ & 0.1 & 0.53 & 0.15 \\
\hline $\begin{array}{l}\text { Polystichum } \\
\text { squarrosum } \\
\text { (D.Don) Fée }\end{array}$ & Dryopteridaceae & Kunji & Yl & $\begin{array}{l}\text { Fresh paste of young leaves used for } \\
\text { curing of fire burns and skin disorders. }\end{array}$ & 0.1 & 0.46 & 0.3 \\
\hline $\begin{array}{l}\text { Equisetum } \\
\text { arvense L. subsp. } \\
\text { arvense }\end{array}$ & Equisetaceae & Bandaky & C & $\begin{array}{l}\text { Decoction of strobilus used for seven } \\
\text { days against bones weakness, arthritis, } \\
\text { osteoporosis, osteomalacia and } \\
\text { vitamin-D deficiency. }\end{array}$ & 0.8 & 0.93 & 0.46 \\
\hline $\begin{array}{l}\text { Equisetum } \\
\text { arvense L. subsp. } \\
\text { diffusum (D.Don) } \\
\text { Frser-Jenk. }\end{array}$ & Equisetaceae & Bandaky & Wp & $\begin{array}{l}\text { Whole Plant powder mixed with cow's } \\
\text { butter and sugar used as an incense to } \\
\text { keep off fear in children. }\end{array}$ & 0.6 & 0.96 & \\
\hline $\begin{array}{l}\text { Equisetum } \\
\text { ramosissimum } \\
\text { Desf. }\end{array}$ & Equisetaceae & Bandaky & C & $\begin{array}{l}\text { Ten grams Strobilus powder mixed with } \\
100 \mathrm{ml} \text { olive oil used in the treatment } \\
\text { of stomach and intestinal disorders, i.e. } \\
\text { ulcer. }\end{array}$ & 0.8 & 0.32 & 0.68 \\
\hline $\begin{array}{l}\text { Hypodematium } \\
\text { crenatum } \\
\text { (Forssk.) Kuhn } \\
\text { subsp. Crenatum }\end{array}$ & Hypodematiaceae & Kunji & $\mathrm{R}$ & $\begin{array}{l}\text { Rhizome decoction used for one month } \\
\text { orally against women infertility and } \\
\text { menstrual disorders. }\end{array}$ & 0.08 & 0.2 & 0.16 \\
\hline $\begin{array}{l}\text { Marsilea minuta } \\
\text { L. }\end{array}$ & Marsileaceae & Yah boty & Yl & $\begin{array}{l}\text { Leaf poultice used as emollient for } \\
\text { treatment of skin problems, i.e. swelling, } \\
\text { irritation, inflammation, itching and } \\
\text { scabies. }\end{array}$ & 0.09 & 0.93 & 0.38 \\
\hline $\begin{array}{l}\text { Adiantum } \\
\text { capillus-veneris L. }\end{array}$ & Pteridaceae & Parshu shah & Yl & $\begin{array}{l}\text { Ten grams of young leaves powder mixed } \\
\text { with } 100 \text { grams of rice and sugar made } \\
\text { into a cake, used against women sterility. }\end{array}$ & 0.1 & 0.4 & 0.46 \\
\hline $\begin{array}{l}\text { Adiantum incisum } \\
\text { Forssk. subsp. } \\
\text { incisum }\end{array}$ & Pteridaceae & Bishushah & Yf & $\begin{array}{l}\text { Young frond } 10 \mathrm{ml} \text { extract mixed } \\
\text { with } 100 \mathrm{ml} \text { mustard oil used against } \\
\text { dandruff, hairs falling,baldness, i.e. } \\
\text { alopecia areata and alopecia. }\end{array}$ & 0.1 & 0.8 & 0.46 \\
\hline $\begin{array}{l}\text { Adiantum } \\
\text { tibeticum Ching }\end{array}$ & Pteridaceae & Parshushah & Yl & $\begin{array}{l}\text { Ten ml young leaves extract mixed with } \\
\text { boiled rice made into a cake used orally } \\
\text { against gastroesophageal reflux, Celiac } \\
\text { disease and gallstones. }\end{array}$ & 0.12 & 0.6 & 0.19 \\
\hline
\end{tabular}

Key for part used: C: Cones; R: Rhizome; WP: Whole plant; Yf: Young frond; Yl: Young leaves. 
Table 1. Continued...

\begin{tabular}{|c|c|c|c|c|c|c|c|}
\hline Taxon & Family & $\begin{array}{l}\text { Vernacular } \\
\text { name }\end{array}$ & $\begin{array}{c}\text { Part } / \mathbf{s} \\
\text { used }\end{array}$ & $\begin{array}{c}\text { Method of application and Disease } \\
\text { cured }\end{array}$ & UV & FL & RFC \\
\hline $\begin{array}{l}\text { Adiantum } \\
\text { venustum D.Don }\end{array}$ & Pteridaceae & Sunrah & Yl & $\begin{array}{l}\text { Ten grams Young leaves grinded powder } \\
\text { mixed with sugar used orally for one } \\
\text { week against common cold, Pertussis and } \\
\text { seasonal influenza. }\end{array}$ & 0.09 & 0.4 & 0.06 \\
\hline $\begin{array}{l}\text { Aleuritopteris } \\
\text { albomarginata } \\
\text { (C.B.Clarke) } \\
\text { Ching }\end{array}$ & Pteridaceae & Jenabil & Yl & $\begin{array}{l}\text { Ten ml young leaves extract mixed } \\
\text { with hot milk used once a day for three } \\
\text { weeks to cure chronic fatigue syndrome, } \\
\text { fibromyalgia and dystonia. }\end{array}$ & 0.08 & 0.94 & 0.07 \\
\hline $\begin{array}{l}\text { Aleuritopteris } \\
\text { formosana } \\
\text { (Hayata) Tagawa }\end{array}$ & Pteridaceae & Jenabil & Yl & $\begin{array}{l}\text { Young leaves extract mixed with honey } \\
\text { used orally against body weakness and } \\
\text { used as a herbal tonic. }\end{array}$ & 0.07 & 0.9 & 0.21 \\
\hline $\begin{array}{l}\text { Aleuritopteris } \\
\text { grisea (Blanf.) } \\
\text { Panigrahi }\end{array}$ & Pteridaceae & Jenabil & Yl & $\begin{array}{l}\text { Ten grams young leaves extract mixed } \\
\text { with cow butter, sugar used to enhance } \\
\text { bones strength and reduce body } \\
\text { weakness. }\end{array}$ & 0.06 & 0.8 & 0.45 \\
\hline $\begin{array}{l}\text { Oeosporangium } \\
\text { nitidulum (Hook.) } \\
\text { Fraser-Jenk. }\end{array}$ & Pteridaceae & Jenbil & Yl & $\begin{array}{l}\text { Leaves extract used for treatment of } \\
\text { cough, disorders of lungs, i.e. Bronchitis, } \\
\text { Asthma and Pneumonia. }\end{array}$ & 0.07 & 0.6 & 0.45 \\
\hline $\begin{array}{l}\text { Oeosporangium } \\
\text { subvillosum } \\
\text { (Hook.) Fraser- } \\
\text { Jenk. \& Pariyar }\end{array}$ & Pteridaceae & Jenabil & Yl & $\begin{array}{l}\text { Ten ml young leaves extract mixed with } \\
100 \mathrm{ml} \text { olive oil used orally as herbal } \\
\text { tonic. }\end{array}$ & 0.1 & 0.53 & 0.26 \\
\hline $\begin{array}{l}\text { Pteris cretica L. } \\
\text { subsp. cretica }\end{array}$ & Pteridaceae & Thandi boty & Yf & $\begin{array}{l}\text { Leaf poultice applied on skin for skin } \\
\text { disorders, swelling, irritation and } \\
\text { inflammation. }\end{array}$ & 0.5 & 0.3 & 0.4 \\
\hline $\begin{array}{l}\text { Pteris vittata L. } \\
\text { subsp. emodi } \\
\text { Fraser Jenk. }\end{array}$ & Pteridaceae & Ghwar boty & Yl & $\begin{array}{l}\text { Young leaves extract mixed with } 100 \\
\text { grams of black cow butter used orally } \\
\text { once a day for one month against } \\
\text { epilepsy. }\end{array}$ & 0.6 & 0.13 & 0.35 \\
\hline $\begin{array}{l}\text { Pteris vittata } \mathrm{L} . \\
\text { subsp. vittata }\end{array}$ & Pteridaceae & Zbarg boty & Yl & $\begin{array}{l}\text { Young leaves paste used for treatment of } \\
\text { body wounds and fire burns. }\end{array}$ & 0.8 & 0.5 & 0.5 \\
\hline $\begin{array}{l}\text { Ampelopteris } \\
\text { prolifera (Retz.) } \\
\text { Copel. }\end{array}$ & Thelypteridaceae & Banjabsa & Yf & $\begin{array}{l}\text { Ten grams young frond extract mixed } \\
\text { with yoghurt used for treatment of } \\
\text { crohn's disease, ulcerative colitis and } \\
\text { irritable bowel syndrome. }\end{array}$ & 0.05 & 0.6 & 0.39 \\
\hline $\begin{array}{l}\text { Christella } \\
\text { dentata (Forssk.) } \\
\text { Brownsey \& } \\
\text { Jermy }\end{array}$ & Thelypteridaceae & Kunji & $\mathrm{R}$ & $\begin{array}{l}\text { Rhizome grinded powder mixed with } \\
\text { coconut oil and sugar used orally against } \\
\text { female infertility. }\end{array}$ & 0.9 & 0.2 & 0.45 \\
\hline $\begin{array}{l}\text { Phegopteris } \\
\text { connectilis } \\
\text { (Michaux) Watt }\end{array}$ & Thelypteridaceae & Banjasa & $\mathrm{R}$ & $\begin{array}{l}\text { Young leaves extract mixed with two } \\
\text { drops of honey and applied in nostrils } \\
\text { twice a day effective in migraine. }\end{array}$ & 0.05 & 0.4 & 0.2 \\
\hline $\begin{array}{l}\text { Pseudophegopteris } \\
\text { levingei } \\
\text { (C.B.Clarke) } \\
\text { Ching }\end{array}$ & Thelypteridaceae & Konji & $\mathrm{R}$ & $\begin{array}{l}\text { Rhizome decoction mixed with olive oil } \\
\text { and applied on ear and nose jewelries } \\
\text { wounds healing. }\end{array}$ & 0.2 & 0.4 & 0.4 \\
\hline $\begin{array}{l}\text { Pseudophegopteris } \\
\text { microstegia } \\
\text { subsp. laterepens } \\
\text { (E.W. Trotter) } \\
\text { Fraser-Jenk. }\end{array}$ & Thelypteridaceae & Jenabil & Yl & $\begin{array}{l}\text { Leaf extract mixed with Memordica } \\
\text { extract used against diabetes mellitus. }\end{array}$ & 0.1 & 0.35 & 0.16 \\
\hline
\end{tabular}

Key for part used: C: Cones; R: Rhizome; WP: Whole plant; Yf: Young frond; Yl: Young leaves. 
Table 1. Continued...

\begin{tabular}{|c|c|c|c|c|c|c|c|}
\hline Taxon & Family & $\begin{array}{l}\text { Vernacular } \\
\text { name }\end{array}$ & $\begin{array}{c}\text { Part } / \mathbf{s} \\
\text { used }\end{array}$ & $\begin{array}{c}\text { Method of application and Disease } \\
\text { cured }\end{array}$ & UV & FL & RFC \\
\hline $\begin{array}{l}\text { Woodsia } \\
\text { glabella R.Br. ex } \\
\text { Richardson }\end{array}$ & Woodsiaceae & Manji boty & Yl & $\begin{array}{l}\text { Young leaves extract used orally in cough } \\
\& \text { cold disorders of lungs, chest, i.e. } \\
\text { Asthma and bronchitis. }\end{array}$ & 0.1 & 0.2 & 0.2 \\
\hline
\end{tabular}

Key for part used: C: Cones; R: Rhizome; WP: Whole plant; Yf: Young frond; Yl: Young leaves.

largest with 12 taxa (30\%), followed by Athyriaceae and Dryopteridaceae with 6 taxa each (30\%), Thelypteridaceae with five (12.5\%), followed by Aspleniaceae presenting 4 (10\%) and Equisetaceae with 3 taxa (7.5\%). Blechnaceae, Hypodematiaceae, Marsileaceae and Woodsiaceae presented 1 taxon each (10\%) (Figure 1, Table 1). Adiantun, Asplenium and Dryopteris were the most representative genera with 4 taxa each (30\%), followed by Aleuritopteris, Diplazium, Pteris and Equisetum were second with 3 taxa each (30\%), Athyrium, Oeosporangium, Polystichum and Pseudophegopteris with 2 taxa each (20\%), and Ampelopteris, Christella, Cystopteris, Hypodematium, Marsilea, Phegopteris, Woodsia and Woodwardia with 1 taxon each (20\%) (Figure 2, Table 1). All of these taxa were used in the treatment of different diseases, i.e. healing of wounds, general tonic, asthma, bronchitis, pneumonia, skin infections, ulcer, sterility in women, diabetes, hepatitis, gonorrhea, common cold, as an anthelmintic, rheumatism, epilepsy and osteoporosis while two taxa of Diplazium were used as a vegetable. Different parts of ferns were used by local inhabitants, i.e. young leaves of twenty one taxa (52.5\%), rhizome of nine taxa (22.5\%), young frond of seven taxa (17.5\%), cones of two taxa (5\%), while whole plant of only one taxa (2.5\%) were used. Seven taxa was used for skin infections (17.5\%), followed by female sterility and general tonic with 4 taxa each (20\%), followed by intestinal disorders and wound healings with 3 taxa each (15\%), lungs, bones, stomach disorders have 2 taxa each (15\%), while remaining disorders, i.e. antivenom, rheumatism, migraine, diabetes, epilepsy and as an expectorant etc have only 1 taxon each (32.5\%) were used for treating them (Table 1). The effectiveness of the treatments described has not been tested, as the present study aimed to rescue and record the knowledge contained in traditional populations regarding ferns and how they are used by these inhabitants. Frequency and percentage of different ethnic groups in the area resulted that Pashto was the dominant language (98\%) followed by Gujars (2\%). Gender wise men had more information about vernacular names and uses of taxa (70\%) followed by women (30\%). Hakims, wound healers, medicinal plant traders and farmers presented more information on the use of these taxa. Locality wise rural and hilly areas (81.33\%) people have more knowledge about the ethnomedicinal uses of taxa, followed by suburban and urban areas (19.67\%).

\subsection{Use value (UV)}

Use value is used to identify the plant species which was used extensively amongst indigenous communities for various ailments. In present study use value of ferns species ranges from $(0.90)$ to $(0.04)$; the highest use value

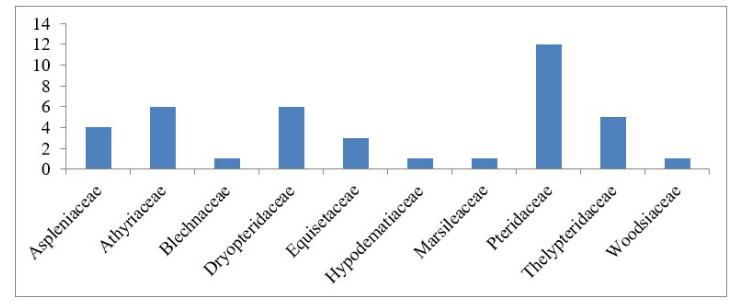

Figure 1. Representation of ethnomedicinal and traditional uses of the ferns families of Khyber Pakhtunkhwa, Pakistan.

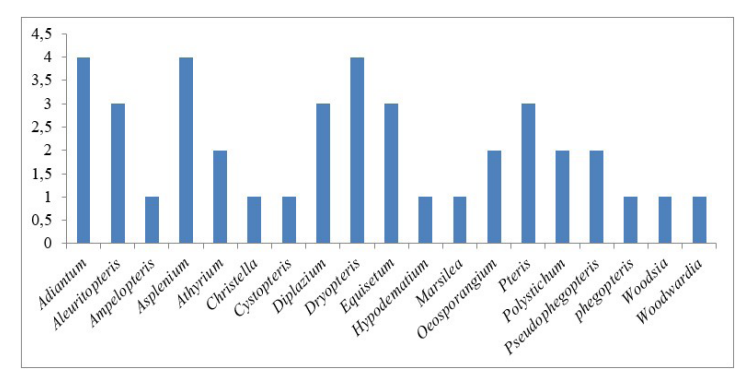

Figure 2. Representation of ethnomedicinal and traditional uses of the ferns genera of Khyber Pakhtunkhwa, Pakistan.

reported were of Athyrium atkinsoni and Christella dentata (0.90) each, followed by Equisetum arvense subsp. arvense, E. ramossimum and Pteris vittata subsp. vittata with (0.80) each, Diplazium longifolium, D. maximum, Equisetum arvense subsp. diffusum and Pteris vittata subsp. emodi (0.60) each, Pteris cretica subsp. cretica (0.50), Diplazium esculentum (0.40), Pseudophegopteris levingei and woodwardia unigemata (0.20) each, Asplenium trichomanes subsp. trichomanes (0.15) and Adiantum tibeticum with (0.12). The species with lowest use value were Dryopteris blandfordi subsp. blanfordi (0.04), Ampelopteris prolifera, Phegopteris connectalis (0.05) each, Aleuritopteris grisea, Asplenium ceterach subsp. ceterach and Dryopteris nigropalacea (0.06) each, Aleuritopteris formosana, Oeosporangium nitidulum (0.07) each, Aleuritopteris albomarginata, Hypodematium crenatum subsp. crenatum and Dryopteris redactopinnata (0.08) each (Table 1$)$.

\subsection{Fidelity level (FL)}

The fidelity level (FL) of ferns species ranged from 0.96 to 0.10 with highest fidelity level (FL) were Equisetum arvense subsp. diffusum (0.96), followed by Aleuritopteris albomarginata (0.94), Equisetum arvense subsp. arvense, 
and Marsilea minuta (0.93) each, Aleuritopteris formosana (0.90) and Adiantum incisum subsp. incisum (0.80) while species with lowest fidelity level (FL) were Athyrium schimperi (0.10), Asplenium adiantum-nigrum subsp. adiantum-nigrum, Pteris vittata subsp. emodi (0.13) each, Woodwardia unigemata, Dryopteris blandfordi subsp. blanfordi, Hypodematium crenatum subsp. crenatum, Christella dentata and Woodsia cycloba (0.20) each, Asplenium trichomanes subsp. trichomanes (0.26), Pteris cretica subsp. cretica (0.30) and Equisetum ramossimum (0.08) (Table 1).

\subsection{Relative frequency of citation (RFC)}

In present study the relative frequency of citation (RFC) ranged from 0.68 to 0.06 with highest value were of Equisetum ramossimum (0.68) followed by Dryopteris stewartii (0.61), Diplazium maximum (0.60), Diplazium longifolium (0.50) and Equisetum arvense subsp. arvense, Adiantum capillus-veneris and Adiantum incisum subsp. incisum (0.46) each; while species with lowest relative frequency of citation (RFC) were Adiantum venusetum (0.06) followed by Aleuritopteris albomarginata (0.07), Woodwardia unigemata (0.10), Polystichum piceopalaceum (0.15), Hypodematium crenatum subsp. crenatum and Pseudophegopteris microstegia subsp. laterpens with (0.16) each (Table 1).

\subsection{Jaccard index (JI)}

Comparison of our study with the previously published articles from India jaccard index (JI) ranged from 5.5 to minus 0.10. The highest Jaccard index (JI) value 5.5, 5.0, 3.33, 3.14 and 2.57 were reported from Rajasthan, India (Parihar and Parihar, 2006); Kolli Hills, Namakkal District,Tamil Nadu, India (Perumal, 2010); Similipal Biosphere Reserve, Orissa, India (Rout et al., 2009) and Vindhyan Region (M.P.) India (Pathak et al., 2011) respectively; while lowest Jaccard index (JI) were -1.0, 0.31, 0.69 and 0.79 were reported from the studies of different parts of India (Kumar et al., 2003); Western Ghats, India (Benjamin and Manickkam, 2007); Banajalaya conserved forest of Shimoga District, Karnataka, India (Deepa et al., 2014) and Arunachal Pradesh, North eastern India (Benniamin, 2011) respectively. Overall the percentage of similar uses ranges from 0.07 to $40.0 \%$ and percentage of dissimilar uses ranges from 0 to $12.50 \%$ (Table 2).

\section{Discussion}

Comparison of our results with previously published articles have highest similarity use index with Kolli hills, Namakkal District, Tamil Nadu, India (Perumal, 2010); Rajasthan, India (Parihar and Parihar, 2006); Kerala, South India (Kumar et al., 2003); Vindhyan Region (M.P.), India (Pathak et al., 2011); Similipal Biosphere Reserve, Orissa,

Table 2. Comparison of given data with previously published articles.

\begin{tabular}{|c|c|c|c|c|c|c|c|c|}
\hline Reported areas & $\begin{array}{c}\text { Total no. } \\
\text { of taxa }\end{array}$ & $\begin{array}{c}\text { Taxa } \\
\text { with } \\
\text { same } \\
\text { uses }\end{array}$ & $\begin{array}{c}\text { Taxa } \\
\text { with } \\
\text { different } \\
\text { uses }\end{array}$ & $\begin{array}{c}\text { Taxa } \\
\text { common } \\
\text { in both } \\
\text { areas }\end{array}$ & $\begin{array}{c}\% \text { of } \\
\text { taxa } \\
\text { with } \\
\text { same } \\
\text { uses }\end{array}$ & $\begin{array}{c}\text { \% of } \\
\text { taxa } \\
\text { with } \\
\text { different } \\
\text { uses }\end{array}$ & JI & Citation \\
\hline $\begin{array}{l}\text { Kumaun Himalaya, } \\
\text { Uttarakhand, India }\end{array}$ & 30 & 04 & 02 & 06 & 13.33 & 6.66 & 2.57 & $\begin{array}{l}\text { Upreti et al. } \\
\text { (2009) }\end{array}$ \\
\hline Different parts of India & 66 & 13 & 05 & 18 & 19.70 & 7.57 & -1.0 & $\begin{array}{l}\text { Kumar et al. } \\
\text { (2003) }\end{array}$ \\
\hline Rajasthan India & 16 & 05 & 02 & 07 & 31.25 & 12.50 & 5.50 & $\begin{array}{l}\text { Parihar and } \\
\text { Parihar } \\
(2006)\end{array}$ \\
\hline $\begin{array}{l}\text { Similipal Biosphere } \\
\text { Reserve, Orissa, India }\end{array}$ & 33 & 06 & 03 & 09 & 18.18 & 9.09 & 3.33 & $\begin{array}{l}\text { Rout et al. } \\
\text { (2009) }\end{array}$ \\
\hline $\begin{array}{l}\text { Arunachal pardesh, North } \\
\text { eastern india }\end{array}$ & 51 & 06 & 02 & 08 & 11.76 & 3.92 & 0.79 & $\begin{array}{l}\text { Benniamin } \\
\text { (2011) }\end{array}$ \\
\hline $\begin{array}{l}\text { Vindhyan Region (M.P.) } \\
\text { India }\end{array}$ & 16 & 03 & 01 & 04 & 18.75 & 6.25 & 3.14 & $\begin{array}{l}\text { Pathak et al. } \\
\text { (2011) }\end{array}$ \\
\hline Western Ghats, India & 57 & 07 & 03 & 10 & 12.28 & 5.26 & 0.31 & $\begin{array}{l}\text { Benjamin } \\
\text { and } \\
\text { Manickkam } \\
(2007)\end{array}$ \\
\hline $\begin{array}{l}\text { Kolli Hills, Namakkal } \\
\text { District,Tamil Nadu, India }\end{array}$ & 10 & 04 & 01 & 05 & 40 & 10 & 5.0 & $\begin{array}{l}\text { Perumal } \\
(2010)\end{array}$ \\
\hline $\begin{array}{l}\text { Banajalaya conserved } \\
\text { forest area of Shimoga } \\
\text { District, Karnataka, India }\end{array}$ & 19 & 01 & 00 & 01 & 5.26 & 00 & 0.69 & $\begin{array}{l}\text { (Deepa et al., } \\
\text { 2014) }\end{array}$ \\
\hline
\end{tabular}

JI: Jaccard index. 
India (Rout et al., 2009); Kumaun Himalaya, Uttarakhand, India (Upreti et al., 2009); Western Ghats, India (Benjamin and Manickkam, 2007); Arunachal Pradesh, North Eastern, India (Benniamin, 2011) and Banajalaya conserved forest area of Shimoga District, Karnataka, India (Deepa et al., 2014), while the lowest JI values were resulted with Kerala, South India (Kumar et al., 2003); Banajalaya conserved forest area of Shimoga District, Karnataka, India (Deepa et al., 2014); Arunachal Pradesh, North Eastern, India (Benniamin, 2011); Kumaun Himalaya, Uttarakhand, India (Upreti et al., 2009); Rajasthan, India (Parihar and Parihar, 2006); Similipal Biosphere Reserve, Orissa, India (Rout et al., 2009) and Kolli hills, Namakkal District, Tamil Nadu, India (Perumal, 2010) respectively (Table 2).

\section{Conclusion}

Ethnomedicinally ferns taxa were utilized by the local inhabitants in the curing of various ailments, i.e. diabetes, hepatitis, hair loss, bones disorders, female infertility, stomach, urinary and intestinal disorders, bronchial disorders, wound healings, general tonic, epilepsy, hair care, inflammation of skin, antivenom, anthelmintic, and fair complexion to treat skin problems with strong potential. These taxa may be utilized commercially for drug discovery, keeping in view of their availability and sustainable supply. Cultivation of these taxa for medicine and ornamental purpose is highly needed. Education plays a key role in awareness about the uses of medicinal plants at scientific basis which can leads to drug discovery.

\section{Acknowledgements}

The authors are grateful to local inhabitants of Khyber Pakhtunkhwa, Pakistan for sharing their valuable indigenous knowledge with us. Special thanks to Mr. C.R. Fraser-Jenkins for identifying collections, providing brief lists of specimens, detailed helpful comments on the typescript and valuable suggestions about the Lycophytes and Ferns of Pakistan. The authors are also grateful to Dr. Li-Bing Zhang and Prof. Dr. Rainer W. Bussmann for their help in writing of manuscript. The first author is grateful for funds received from Higher education commission of Pakistan as an International Ph.D. scholar. The authors also extend their appreciation to the Deanship of Scientific Research at King Khalid University for funding this work through Research group Project under grant number (R.G.P.1/39/42).

\section{References}

AHMAD, M., SULTANA, S., FAZL-I-HADI, S., BEN-HADDA, T., RASHID, S., ZAFAR, M., KHAN, M.A., KHAN, M.P.Z. and YASEEN, G., 2014. An ethnobotanical study of medicinal plants in high mountainous region of Chail valley (District swat, Pakistan). Journal of Ehnobiology and Ethnomedicine, vol. 10, no. 36, pp. 1-18. https://doi.org/10.1186/1746-4269-10-36.

AHMAD, I., IRFAN, M., ALI, I., KHAN, J., SAEED, S.H. and GULFARAZ, A., 2016. Checklist of some medicinal plants of district Lower
Dir, Pakistan. IASET: Journal of Agricultural and Bio-Chemical Science, vol. 1, pp. 15-22.

ALEXIADES, M.N., 1996. Collecting ethnobotanical data: an introduction to basic concepts and techniques. In: M.N. ALEXIADES, editor. Advances in economic botany. New York: The New York Botanical Garden Press, vol. 10, pp. 53-94.

ALI, A., RASHID, M., SULTAN, A. and IRFAN, M., 2017. Anisochilus carnosus (L. f.) Wall. ex Benth. (Lamiaceae) A new generic record for Pakistan. Plant Science Today, vol. 4, no. 3, pp. 102105. http://dx.doi.org/10.14719/pst.2017.4.3.316.

ALI-SHTAYEH, M.S., JAMOUS, R.M. and JAMOUS, R.M., 2016. Traditional Arabic Palestinian ethnoveterinary practices in animal health care: a field survey in the West Bank (Palestine). Journal of Ethnopharmacology, vol. 182, pp. 35-49. http://dx.doi. org/10.1016/j.jep.2016.02.005. PMid:26869545.

ATTAULLAH, KHAN, Z-U.D. and MIDRARULLAH, 2017. Taxonomic and ethnobotanic study of pteridophytes of Miandam valley, District Swat, Khyber Pakhtunkhwa, Pakistan. International Journal of Biosciences, vol. 10, no. 3, pp.72-79.

BENJAMIN, A. and MANICKKAM, V.S., 2007. Medicinal Pteridophytes from Western Ghats. Indian Journal of Traditional Knowledge, vol. 6, no. 4, pp. 611-618.

BENNIAMIN, A., 2011. Medicinal ferns of North Eastern India with special reference to Arunachal Pradesh. Indian Journal of Traditional Knowledge, vol. 10, no. 3, pp. 516-522.

BIPLAB, P. and SUBIR, B., 2007. Herbivore Damage to Ferns Caused by a Chrysomelid Beetle from Lower Gangetic Plains of West Bengal, India. American Fern Journal, vol. 97, no. 1, pp. 19-29. http://dx.doi.org/10.1640/0002-8444(2007)97[19:HDTFCB ]2.0.CO;2.

DEEPA, J., ASHWINI, S. and PARASHURAMA, T.R., 2014. Enumeration of pteridophytes in Banajalaya conserved forest area of Shimoga District, Karnataka. Asian Pacific Journal of Health Sciences, vol. 1, no. 4, pp. 448-450. http://dx.doi.org/10.21276/apjhs.2014.1.4.23.

FRASER-JENKINS, C.R., 1992. The ferns and allies of the far west Himalaya. Pakistan Systematics, vol. 5, pp. 85-120.

FRASER-JENKINS, C.R., 2014. Ferns and Allies of the far-west IndoHimalaya (Afghanistan, Pakistan and Kashmir) and Iran-revised checklists, classification and phytogeography. Indian Fern Journal, vol. 30, pp. 161-191.

FRASER-JENKINS, C.R., GANDHI, K.N. and KHOLIA, B.S. 2018. An Annotated Checklist of Indian Pteridophytes, Part-II, Woodsiaceae to Dryopteridaceae. Dehradun: Bisen Singh Mahendra Pal Singh, pp. 573.

FRASER-JENKINS, C.R., GANDHI, K.N., KHOLIA, B.S. and BENNIAMIN, A., 2016. An annotated checklist of Indian Pteridophytes, Part-I (Lycopodiaceae to Thelypteridace). Dehradun: Bisen Singh Mahendra Pal Singh, pp. 562.

FRASER-JENKINS, C.R., GANDHI, K.N., KHOLIA, B.S. and KANDEL, D.R., 2020. An Annotated Checklist of Indian Pteridophytes, PartIII, Lomariopsidaceae to Salviniaceae. Dehradun: Bisen Singh Mahendra Pal Singh, pp. 420.

GONZÁLEZ-TEJERO, M.R., CASARES-PORCEL, M., SÁNCHEZ-ROJAS, C.P., RAMIRO-GUTIÉRREZ, J.M., MOLERO-MESA, J., PIERONI, A., GIUSTI, M.E., CENSORII, E., DE PASQUALE, C., DELLA, A., PARASKEVA-HADIJCHAMBI, D., HADJICHAMBIS, A., HOUMANI, Z., EL-DEMERDASH, M., EL-ZAYAT, M., HMAMOUCHI, M. and ELJOHRIG, S., 2008. Medicinal plants in the Mediterranean area: synthesis of the results of the project Rubia. Journal of Ethnopharmacology, vol. 116, no. 2, pp. 341-357. http://dx.doi. org/10.1016/j.jep.2007.11.045. PMid:18242025.

GUL, A., ALAM, J., AHMAD, H. and IRFAN, M., 2016a. An updated checklist of Pteridophytes of district Mansehra, Khyber 
Pukhtunkhwa-Pakistan. Plant Science Today, vol. 3, no. 2, pp. 237-247. http://dx.doi.org/10.14719/pst.2016.3.2.220.

GUL, A., ALAM, J., AHMAD, H., SHAH, G.M., HUSSAIN, M., DOGAN, Y. and RAHMAN, K.U., 2016b. Traditional medicinal and food uses of Pteridophytes of district Mansehra and their some adjacent areas. International Journal of Biosciences, vol. 9, no. 5, pp. 116-133. http://dx.doi.org/10.12692/ijb/9.5.116-133.

IFTIKHAR, S., ALI, W., ULLAH, S., KHAN, W. and IRFAN, M., 2019. Comparative antibacterial potential of methanolic extract of the leaves of wild and cultivated Ficus carica L. International Journal of Botany Studies, vol. 4, no. 4, pp. 139-143.

IRFAN, M., AHMAD, I. and SAEED, S.H., 2017a. Traditional medicinal plant knowledge of some spermatophytes of Samar Bagh Valley, Lower Dir district, Pakistan. Plant Science Today, vol. 4 , no. 4, pp. 151-153. http://dx.doi.org/10.14719/pst.2017.4.4.334.

IRFAN, M., ALI, D., JAN, G. and MURAD, W., 2018a. Ethnobotanical Survey of the Flora of Tehsil Balakot, District Mansehra, Khyber Pakhtunkhwa, Pakistan. Science Arena Publications Specialty Journal of Biological Sciences, vol. 4, no. 3, pp. 7-14.

IRFAN, M., ALI, I. and KASHIF, R.A., 2018b. Ethnobotanical survey of the flora of Maidan Valley, Lower Dir District, Khyber Pakhtunkhwa Province, Pakistan. Plant Science Today, vol. 5, no. 2, pp. 68-71. http://dx.doi.org/10.14719/pst.2018.5.2.379.

IRFAN, M., JAN, G., JAN, F.G. and MURAD, W., 2021a. A taxonomic revision of the genus Selaginella (Selaginellaceae: Lycopodiopsida) from Pakistan. The Journal of Animal and Plant Sciences, vol.31, no. 3, pp. 888-893. http://dx.doi.org/10.36899/ JAPS.2021.3.0277.

IRFAN, M., JAN, G., JAN, F.G. and MURAD, W., 2021b. Taxonomy and spore morphology of genus Adiantum (Vittarioideae; Pteridaceae) from Pakistan. Microscopy Research and Technique vol. 84, no. 11, pp. 2727-2736. http://dx.doi.org/10.1002/ jemt.23835. PMid:34051004.

IRFAN, M., JAN, G., JAN, F.G. and MURAD, W., 2021c. Taxonomy and spore morphology of selected taxa of Cheilanthoideae and Pteridoideae (Pteridaceae) from Pakistan. Microscopy Research and Technique, vol. 84, no. 12, pp. 2867-2882. http://dx.doi. org/10.1002/jemt.23845. PMid:34121271.

IRFAN, M.,JAN, G., JAN, F.G. and MURAD, W., 2021d. Floristic diversity and chorotype analysis of the pteridophytes of Pakistan. The Journal of Animal and Plant Sciences, vol. 32, no. 1, pp. 1-14. http://dx.doi.org/10.36899/JAPS.2022.1.0395.

IRFAN, M., KHAN, I., ALI, A., KHAN, R., ALI, A. and JAN, G., 2017b. Ethnomedicinal Uses of the Plants of Tehsil Laalqilla, District Lower Dir, Khyber Pakhtunkhwa, Pakistan. Journal of Applied Environmental \& Biological Sciences, vol. 8, no. 6, pp. 61-66.

IRFAN, M., NABEELA, Kamil, M., KHAN, N.A., ALI, A., ULLAH, Z., ILYAS, M., AHMAD, I., ALI, A., ULLAH, S., SUBHAN, F. and KHAN, U., 2018c. Ethnomedicinal applications of plant taxa by the local communities of Tehsil Adenzai, District Lower Dir, Khyber Pakhtunkhwa, Pakistan. International Journal of Biosciences, vol. 13, no. 5, pp. 40-49. http://dx.doi.org/10.12692/ijb/13.5.40-49.

IRFAN, M., NABEELA, KAMIL, M., KHAN, N.A., ILYAS, M., ALI, A., ULLAH, S., SHAH, M., JAN, G. and MURAD,W., 2018d. Ethomedicinal and traditional knowledge of phanerogames of Tehsil Munda, district Lower Dir, Khyber Pakhtunkhwa, Pakistan. International Journal of Biosciences, vol. 13, no. 4, pp. 208-218. http://dx.doi.org/10.12692/ijb/13.4.208-218.

IRFAN, M., NABEELA, KAMIL, M., KHAN, N.A., KHAN, H., KHALIL, S., ULLAH, S., SHAH, M., JAN, G. and MURAD, W., 2018e. Ethnomedicinal plants uses of tehsil Khall, district Lower Dir, Khyber Pakhtunkhwa, Pakistan. International Journal of
Biosciences, vol. 13, no. 4, pp. 219-229. http://dx.doi.org/10.12692/ ijb/13.4.219-229.

IRFAN, M., NABEELA, KHAN, H. and KHAN, S., 2019. A review of different phytochemicals and pharmacological activities evaluations of Morus alba L. Specialty Journal of Chemistry, vol. 4, no. 2, pp. 1-9.

KAUSHIK, P. and DHIMAN, K., 1995. Common medicinal Pteridophytes. Indian Fern Journal, vol. 12, pp. 139-145.

KAYANI, S., AHMAD, M., ZAFAR, M., SULTANA, S., KHAN, M.P.Z., ASHRAF, M.A., HUSSAIN, J. and YASEEN, G., 2014. Ethnobotanical uses of medicinal plants for respiratory disorders among the inhabitants of Gallies-Abbottabad, Northern Pakistan. Journal of Ethnopharmacology, vol. 156, pp. 47-60.

KELLER, H.A. and PRANCE, G.T., 2015. The ethnobotany of Ferns and Lycophytes. The Fern Gazette, vol. 20, pp. 1-13.

KELLER, H.A., TORRES, E.I.M. and PRANCE, G.T., 2011. Ethnopteridology of the Guaranís of Misiones Province, Argentina. American Fern Journal, vol. 101, no. 3, pp. 193-204. http://dx.doi. org/10.1640/0002-8444-101.3.193.

KUMAR, M., REMESH, M. and SEQUIERA, S., 2003. Medicinal pteridophytes of Kerala, South India. Indian Fern Journal, vol. 20, pp. 1-28.

LIU, Y., WUJISGULENG, W. and LONG, C., 2012. Food uses of ferns in China: a review. Acta Societatis Botanicorum Poloniae, vol. 81, no. 4, pp. 263-270. http://dx.doi.org/10.5586/asbp.2012.046.

MANICKAM, V.S. and IRUDAYARAJ, V., 2003. Pteridophyte Flora of Nilgiris, South India. Dehradun: Bisen Singh Mahendra Pal Singh.

MANNAN, M.M., MARIDASS, M. and VICTOR, B., 2008. A review on the potential uses of Ferns. Ethnobotanical Leaflets, vol. 12, pp. 281-285.

MAY, L.W., 1978. The economic uses and associated folklore of ferns and famallies. Botanical Review, vol. 44, no. 4, pp. 491528. http://dx.doi.org/10.1007/BF02860848.

MITTAL, T.C. and BIR, S.S., 2006. The Indian substitutes of Male Fem Dryopteris filix-mas (L.) Schott Taxonomy and drug macroscopy. Indian Fern Journal, vol. 23, pp. 133-187.

NAIR, B.K., 1959. Medicinal Ferns of India. Bulletin of National Botanical Garden Lucknow, vol. 29, pp. 1-36.

OLOYEDE, F.A., ALAFE, B.O. and OLOYEDE, F.M., 2008. Nutrient evaluation of Nephrolepis bisserata (Nephrolepidiaceae, Pteridophyta). Botanica Lithuanica, vol. 14, no. 4, pp. 207-210.

PANDE, P.C., 1991. An Illustrated Fern Flora of Kumaun Himalaya. Dehradun: Bisen Singh Mahendra Pal Singh. Vol. 1, 2.

PARIHAR, P. and PARIHAR, L., 2006. Some Pteridophytes of medicinal importance from Rajasthan, India. Natural Product Radiance, vol. 5, no. 4, pp. 297-301.

PATHAK, A., SINGH, A. and SINGH, A.P., 2011. Ethnomedicinal uses of pteridophytes of Vindhyan Region (M.P.). International Journal of Pharmacy and Life Sciences, vol. 1, no. 2, pp. 496-498.

PERUMAL, G., 2010. Ethnomedicinal Use of Pteridophyte from Kolli Hills, Namakkal District, Tamil Nadu,India. Ethnobotanical Leaflets, vol. 14, pp. 161-172.

PHILLIPS, O.L., HALL, P., GENTRY, A.H., SAWYER, S.A. and VASQUEZ, R., 1994. Dynamics and species richness of tropical forests. Proceedings of the National Academy of Sciences of the United States of America, vol. 91, no. 7, pp. 2805-2809. http://dx.doi. org/10.1073/pnas.91.7.2805. PMid:11607468.

PPG- I, 2016. A community-derived classification for extant lycophytes and ferns. Journal of Systematics and Evolution, vol. 54, no. 6, pp. 563-603. http://dx.doi.org/10.1111/jse.12229.

RANIL, R.H.G. and BUSSMANN, R.W., 2021. Potential uses of Lycophytes and Ferns in Sri Lanka: an ethnopteridological 
perspective. Ethnobotany Research and Applications, vol. 21, no. 36, pp. 1-11. http://dx.doi.org/10.32859/era.21.36.1-11.

RANIL, R.H.G., BENERAGAMA, C.K., PUSHPAKUMARA, D.K.N.G. and WIJESUNDARA, D.S.A., 2015. Ornamental Pteridophytes: an underexploited opportunity for the Sri Lankan floriculture industry. Journal of the National Science Foundation of Sri Lanka, vol. 43, no. 4, pp. 293-301. http://dx.doi.org/10.4038/ jnsfsr.v43i4.7964.

ROUT, S.D., PANDA, T. and MISHRA, N., 2009. Ethnomedicinal studies on some pteridophytes of similipal biosphere reserve, Orissa, India. International Journal of Medicine and Medical Sciences, vol. 1, no. 5, pp. 192-197.

SINGH, B.P. and UPADHYAY, R., 2014. Medicinal Pteridophytes of Madhya Pradesh. Journal of medicinal plants studies, vol. 2, no. 4, pp. 65-68.
SINGH, H.B. and VISWANATHAN, M.V., 1996. Useful Pteridophytes of India: a gift of nature to human beings. Journal of Economic and Taxonomic Botany, vol. 12, pp. 24-36.

ULLAH, S., KHAN, W., ALI, W., KHAN, M.S., SAJAD, M.A., NABEELA, AND IRFAN, M., 2018. Antibacterial and antifungal potentials of the various solvents extracts of Quercus incana fruits. International Journal of Biosciences, vol. 13, no. 5, pp. 438-447. http://dx.doi.org/10.12692/ijb/13.5.438-447.

UPRETI, K., JALAL, J.S., TEWARI, L.M., JOSHI, G.C., PANGTEY, Y.P.S. and TEWARI, G., 2009. Ethnomedicinal uses of Pteridophytes of Kumaun Himalaya, Uttarakhand, India. The Journal of American Science, vol. 5, no. 4, pp. 167-170.

VASUDEV, S.M., 1999. Economic importance of Pteridophytes. Indian Fern Journal, vol. 16, no. 2, pp. 130-152. 